\title{
Principles of Quantitation: Chromatography
}

\author{
Lane C. Sander \\ National Institute of Standards and Technology, \\ Gaithersburg, MD 20899, USA \\ lane.sander@nist.gov
}

Video DOI: http://doi.org/10.18434/T4DK5T

Key words: calibration; chromatography; internal standards; quantitation; quantitative analysis; response factor.

Accepted: December 1, 2016

Published: January 12, 2017

https://doi.org/10.6028/jres.122.005

\section{Summary}

The accurate characterization of the chemical composition of a sample depends on a variety of factors that must be considered by the analyst. Knowledge of how different approaches to quantitation affect the measurement accuracy is requisite to the development of methods of analysis. This presentation will describe some of the principles of quantitative analysis, particularly as applicable to chromatographic methods. Differences between the external standard calibration approach, internal standard calibration approach, and standard addition are detailed and advantages and disadvantages of the three approaches are provided. Different types of calibration models are explained. Most commonly, calculations are based on averaged response factors, linear regression with either zero intercept or linear regression with calculated intercept. The selection of an appropriate model should be based on knowledge of the measurement system - such as the anticipated analyte levels, matrix interferences, noise, drift, etc.

Other factors affect the accuracy of measurements. The design of a measurement protocol should include consideration of all factors that contribute to uncertainty. Discussion is provided on sample handling, the addition of internal standards, the number of calibrants, the number of replicate measurements, sample queue design, drift and inhomogeneity, integration of partially separated components, use of controls, and measurement traceability. It is concluded that the practice of higher order chemical metrology requires a significant commitment by the analyst: knowledge of measurement principles, laboratory skills, implementation of technology, and attention to detail. ${ }^{1}$

\footnotetext{
${ }^{1}$ Contribution of the National Institute of Standards and Technology. Not subject to copyright. Certain commercial equipment, instruments, or materials are identified to specify adequately the experimental procedure. Such identification does not imply recommendation or endorsement by the National Institute of Standards and Technology, nor does it imply that the materials or equipment identified are the best available for the purpose.
} 